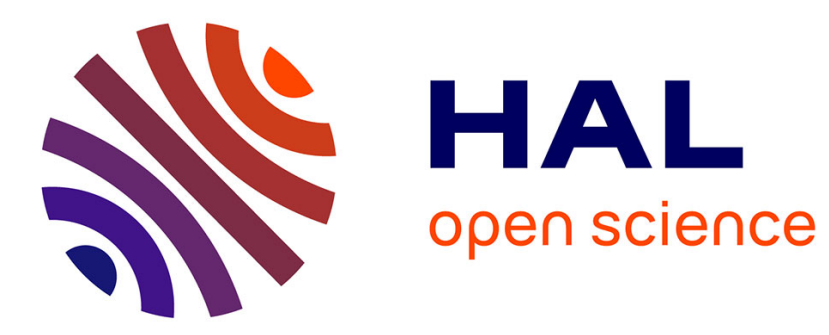

\title{
From Euclidean triangles to the hyperbolic plane
} Ismail Saglam

\section{To cite this version:}

Ismail Saglam. From Euclidean triangles to the hyperbolic plane. Expositiones Mathematicae, In press, 10.1016/j.exmath.2021.10.003 . hal-03419775

\section{HAL Id: hal-03419775 \\ https://hal.science/hal-03419775}

Submitted on 8 Nov 2021

HAL is a multi-disciplinary open access archive for the deposit and dissemination of scientific research documents, whether they are published or not. The documents may come from teaching and research institutions in France or abroad, or from public or private research centers.
L'archive ouverte pluridisciplinaire HAL, est destinée au dépôt et à la diffusion de documents scientifiques de niveau recherche, publiés ou non, émanant des établissements d'enseignement et de recherche français ou étrangers, des laboratoires publics ou privés. 


\title{
From Euclidean triangles to the hyperbolic plane
}

\author{
Ismail Saglam * \\ Adana Alparslan Turkes Science and Technology University, \\ Institut de Recherche Mathematique Avancée, CNRS et \\ Université de Strasbourg
}

\begin{abstract}
We introduce a model of the hyperbolic plane which arises from Thurston's ideas on best Lipschitz maps between surfaces. More precisely, we apply Thurston's theory to the space of Euclidean triangles. We prove that the Lipschitz constant of the unique affine map between two Euclidean triangles induces a metric on the set of isometry classes of the triangles with area $\frac{1}{2}$. We then prove that this space of triangles together with 2 times its natural metric is isometric to the hyperbolic plane. Our construction is simple and natural and it makes relations between several geometrical ideas. This paper will appear in the journal Expositiones Mathematicae.
\end{abstract}

Keywords: Euclidean triangles, Lipschitz maps, hyperbolic plane

\section{Introduction}

Our aim in this paper is to introduce a model for the hyperbolic plane arising from Thurston's ideas on best Lipschitz maps between hyperbolic surfaces,

*Electronic address: isaglamtrfr@gmail.com

2000 Mathematics Subject Classification: 51F99, 57M50 
which he developed in his preprint [2], and which we adapt to a Euclidean setting. Let us first recall some basic facts from Thurston's theory.

Let $S$ and $S^{\prime}$ be two hyperbolic surfaces and $\phi: S \rightarrow S^{\prime}$ be a homeomorphism. Thurston defines

$$
L(\phi)=\sup _{x \neq y}\left\{\frac{d_{S^{\prime}}(\phi(x), \phi(y))}{d_{S}(x, y)}\right\} .
$$

Thurston is interested in the following question: What is the least value of $L(\phi)$ for a homeomorphism $\phi^{\prime}$ in the homotopy class of $\phi$ ? This question naturally gives rise to a metric on Teichmüller space of a given surface as follows. Let $M^{2}$ be a surface of finite type. If $g$ and $h$ are two complete hyperbolic structures on $M^{2}$ representing two elements in the Teichmüller space of $M^{2}$, Thurston defines

$$
L(g, h)=\inf _{\phi \equiv i d}(\log (L(\phi))) .
$$

where in computing $L(\phi)$, the metric $g$ is used before $\phi$ and the metric $h$ is used after $\phi$. Note that $\phi \equiv i d$ means that $\phi$ is homotopic to the identity map of $M^{2}$. Thurston showed that this definition equips the Teichmüller space of the surface with an asymmetric metric. We recall that an asymmetric metric on a set $X$ is a function $\eta: X \times X \rightarrow \mathbb{R}$ which satisfies all the axioms of a metric except the symmetry axiom.

We now introduce the main result of the present paper. Consider two triangles in the Euclidean plane. Label their vertices with the set $\{1,2,3\}$ so that the labeling induces a counter-clockwise orientation on the boundary of each triangle. There is a unique label-preserving affine map which sends one of the triangles to the other one. We calculate the Lipschitz constant of this affine map and we show that this Lipschitz constant induces a metric on the set of equivalence classes of labeled triangles with area $\frac{1}{2}$, where two triangles are equivalent if there is a label preserving isometry between them.

\section{The Lipschitz constant of a linear map}

Let $\left(X_{1}, d_{1}\right)$ and $\left(X_{2}, d_{2}\right)$ be two metric spaces and $f$ be a map between them. We define the Lipschitz constant of the map $f$ to be

$$
L(f)=\sup _{x, y \in X_{1}, x \neq y}\left\{\frac{d_{2}(f(x), f(y))}{d_{1}(x, y)}\right\} .
$$


Consider the Euclidean metric on $\mathbb{R}^{2}$ and let $f: \mathbb{R}^{2} \rightarrow \mathbb{R}^{2}$ be a linear map. If we identify $\mathbb{R}^{2}$ with $\mathbb{C}$, then $f$ can be written as

$$
f(z)=\alpha z+\beta \bar{z},
$$

where $\alpha, \beta \in \mathbb{C}$. In that case, it is known that

$$
L(f)=|\alpha|+|\beta|
$$

(see [1]).

\section{$3 \quad$ Space of triangles}

A labeled triangle is a triangle in the Euclidean plane whose vertices are labeled with the set $\{1,2,3\}$ so that the labeling induces a counter-clockwise orientation on the boundary of the triangle. Denote the set of isometry classes of the labeled triangles having area $\frac{1}{2}$ by $\mathfrak{T}$. The isometries considered are assumed to be label preserving. Consider the upper half-plane

$$
\mathbb{H}=\{z=x+\imath y \in \mathbb{C}: x, y \in \mathbb{R}, y>0\} .
$$

$\mathfrak{T}$ can be identified with $\mathbb{H}$ as follows. Each element in $\mathfrak{T}$ has a unique representative triangle $T$ so that

1. the vertex of $T$ with label 1 is at the origin,

2. the vertex of $T$ with label 2 is on the set of positive numbers,

3. the vertex of $T$ with label 3 is on $\mathbb{H}$.

This implies that $T$ is a triangle with the vertex set $\left\{0, \frac{1}{y}, x+\imath y\right\}$, where $x, y \in \mathbb{R}$ and $y>0$. Conversely, if $x+\imath y \in \mathbb{H}$, then the triangle with vertices $\left\{0, \frac{1}{y}, x+\imath y\right\}$ gives an element in $\mathfrak{T}$.

\section{Calculation of the Lipschitz constant of the linear map between two triangles}

Let $x_{1}+\imath y_{1}$ and $x_{2}+\imath y_{2}$ be two elements in $\mathbb{H}$. Consider the two triangles that these two elements represent. There is a unique label-preserving linear 
map $\phi$ which sends the vertices of the first triangle to the vertices of the second one. It is obtained by setting

1. $\phi(0)=0$,

2. $\phi\left(\frac{1}{y_{1}}\right)=\frac{1}{y_{2}}$,

3. $\phi\left(x_{1}+\imath y_{1}\right)=x_{2}+\imath y_{2}$.

With respect to the standard basis of $\mathbb{R}^{2}, \phi$ has the following matrix representation:

$$
\left(\begin{array}{ll}
a & b \\
c & d
\end{array}\right)=\left(\begin{array}{cc}
\frac{y_{1}}{y_{2}} & \frac{x_{2}}{y_{1}}-\frac{x_{1}}{y_{2}} \\
0 & \frac{y_{2}}{y_{1}}
\end{array}\right)
$$

In complex notation, $\phi(z)=\alpha z+\beta \bar{z}$, where

$$
\begin{aligned}
& \alpha=\frac{a+\imath c-(b+\imath d) \imath}{2}, \\
& \beta=\frac{a+\imath c+(b+\imath d) \imath}{2}
\end{aligned}
$$

Therefore we have

$$
\begin{aligned}
& \alpha=\frac{\frac{y_{1}}{y_{2}}+\frac{y_{2}}{y_{1}}-\imath\left(\frac{x_{2}}{y_{1}}-\frac{x_{1}}{y_{2}}\right)}{2} \\
& \beta=\frac{\frac{y_{1}}{y_{2}}-\frac{y_{2}}{y_{1}}+\imath\left(\frac{x_{2}}{y_{1}}-\frac{x_{1}}{y_{2}}\right)}{2}
\end{aligned}
$$

It follows that

$$
\begin{gathered}
L(\phi)=\frac{1}{2} \sqrt{\left(\frac{y_{1}}{y_{2}}+\frac{y_{2}}{y_{1}}\right)^{2}+\left(\frac{x_{2}}{y_{1}}-\frac{x_{1}}{y_{2}}\right)^{2}}+\frac{1}{2} \sqrt{\left(\frac{y_{1}}{y_{2}}-\frac{y_{2}}{y_{1}}\right)^{2}+\left(\frac{x_{2}}{y_{1}}-\frac{x_{1}}{y_{2}}\right)^{2}} \\
=\frac{\sqrt{\left(x_{1} y_{1}-x_{2} y_{2}\right)^{2}+\left(y_{1}^{2}+y_{2}^{2}\right)^{2}}+\sqrt{\left(x_{1} y_{1}-x_{2} y_{2}\right)^{2}+\left(y_{1}^{2}-y_{2}^{2}\right)^{2}}}{2 y_{1} y_{2}}
\end{gathered}
$$

We now define a function $d$ on $\mathbb{H} \times \mathbb{H}$ by:

$$
=\log \left(\frac{d\left(x_{1}+\imath y_{1}, x_{2}+\imath y_{2}\right)=\log L(\phi)}{2 y_{1} y_{2}}\right)
$$




\section{Relation with the hyperbolic plane}

Let $w_{1}=u_{1}+\imath v_{1}, w_{2}=u_{2}+\imath v_{2} \in \mathbb{H}$. It is well-known that the hyperbolic metric on $\mathbb{H}$ is given by the following formula:

$$
d_{\mathbb{H}}\left(w_{1}, w_{2}\right)=2 \log \left(\frac{\sqrt{\left(u_{2}-u_{1}\right)^{2}+\left(v_{2}-v_{1}\right)^{2}}+\sqrt{\left(u_{2}-u_{1}\right)^{2}+\left(v_{2}+v_{1}\right)^{2}}}{2 \sqrt{v_{1} v_{2}}}\right)
$$

Theorem 1. The function d gives a metric on $\mathbb{H}$. The metric spaces $(\mathbb{H}, 2 d)$ and $\left(\mathbb{H}, d_{\mathbb{H}}\right)$ are isometric.

Proof. To prove both statements of the theorem, it is enough to show that the map $\mathcal{A}: \mathbb{H} \rightarrow \mathbb{H}$ given by

$$
\mathcal{A}(x+\imath y)=x y+\imath y^{2}
$$

has the property

$$
d_{\mathbb{H}}\left(\mathcal{A}\left(\zeta_{1}\right), \mathcal{A}\left(z_{2}\right)\right)=d\left(\zeta_{1}, \zeta_{2}\right)
$$

for all $\zeta_{1}, \zeta_{2} \in \mathbb{H}$.

If $\zeta_{1}=x_{1}+\imath y_{1}$ and $\zeta_{2}=x_{2}+\imath y_{2}$, then $\mathcal{A}\left(\zeta_{1}\right)=w_{1}=x_{1} y_{1}+\imath y_{1}^{2}$ and $\mathcal{A}\left(\zeta_{2}\right)=x_{2} y_{2}+\imath y_{2}^{2}$. Then,

$d_{\mathbb{H}}\left(\mathcal{A}\left(\zeta_{1}\right), \mathcal{A}\left(\zeta_{2}\right)\right)$

$$
=2 \log \left(\frac{\sqrt{\left(x_{2} y_{2}-x_{1} y_{1}\right)^{2}+\left(y_{2}^{2}-y_{1}^{2}\right)}+\sqrt{\left(x_{2} y_{2}-x_{1} y_{1}\right)^{2}+\left(y_{1}^{2}+y_{2}^{2}\right)}}{2 \sqrt{y_{1}^{2} y_{2}^{2}}}\right)
$$

$=2 d\left(\zeta_{1}, \zeta_{2}\right)$

\section{Acknowledgments}

The question addressed in the present paper is suggested by Athanase Papadopoulos. The author is grateful to him for his remarks and comments. This work is supported by TÜBİTAK. 


\section{References}

[1] Belkhirat, A., Papadopoulos, A., Troyanov, M. (2005). Thurston's weak metric on the Teichmüller space of the torus. Transactions of the American Mathematical Society, 357(8), 3311-3324.

[2] Thurston, W. P. (1998). Minimal stretch maps between hyperbolic surfaces. arXiv preprint math/9801039 . 